\title{
Acknowledgement of Reviewers for 2020
}

\author{
Ranjith Pathegama Gamage
}

Accepted: 11 January 2021/Published online: 30 January 2021

(C) Crown 2021

The Editors-in-Chief and the Publisher wish to acknowledge the assistance of the following colleagues, scientists across the world helping us to review articles for our Journal in tight timeframes during the year of 2020. The Editors-in-Chief are very grateful for all the reviewers for providing in-depth review reports for authors to improve the quality of the scripts.

P. Abbasi Maedeh

A. Abbaszadeh Shahri

I. Abdulagatov

F. Abija

W. Akande

M. Alam

W. Alameri

A. Amirlatifi

B. Badenes

A. Bandara

C. Cao

P. Carrubba

S. Chai

D. Chandrasekharam

A. Chaudhuri

X. Chen

Z. Chu

J. Daemen

Z. Dai

S. den Hartog
R. DeSilva

F. Du

M. Everett

L. Fan

Y. Fang

O. Gaede

Q. Gan

M. Gao

N. Gershenzon

L. Gong

H. Güneyli

J. Guo

Z. Guo

M. He

C. Hongran

Z. Hou

X. Hu

D. Huang

F. Huang

S. Huang
Z. Huang

Y. Jia

Q. H. Jiang

H. Jing

Y. Ju

O. Kavak

X. Kong

P. Kundu

J. Larrahondo

B. $\mathrm{Li}$

C. $\mathrm{Li}$

D. $\mathrm{Li}$

J. Li

L. $\mathrm{Li}$

Y. Li

Z. $\mathrm{Li}$

L. Lichuanqi

H. Liu

J. Liu

S. Liu

R. Pathegama Gamage ( $\square)$

Monash University, Clayton, VIC, Australia

e-mail: ranjith.pg@monash.edu 


$\begin{array}{lll}\text { X. Liu } & \text { Y. Shen } & \text { Z. Wu } \\ \text { Y. Liu } & \text { S. Song } & \text { J. Xie } \\ \text { Z. Liu } & \text { Z. Song } & \text { J. Xu } \\ \text { A. Liyanage } & \text { C. Spiers } & \text { T. Xu } \\ \text { Q. Lyu } & \text { Q. Sun } & \text { Y. Xue } \\ \text { K. Ma } & \text { W. Sun } & \text { S. Yang } \\ \text { B. Mahanta } & \text { I. Takuya } & \text { W. Yang } \\ \text { F. Mainardi } & \text { M. Tang } & \text { J. Yu } \\ \text { K. Min } & \text { Z. Tang } & \text { Wei Yu } \\ \text { M. Mohammadnejad } & \text { D. Wang } & \text { Y. Yu } \\ \text { R. Nair } & \text { H. Wang } & \text { N. Zakharova } \\ \text { M. Nasvi } & \text { K. Wang } & \text { Z. Zeng } \\ \text { P. Ni } & \text { S. Wang } & \text { C. Zhang } \\ \text { B. Noetinger } & \text { Y. Wang } & \text { D. Zhang } \\ \text { B. Novac } & \text { Z. Wang } & \text { F. Zhang } \\ \text { H. Ozturk } & \text { M. Wangen } & \text { G. Zhang } \\ \text { S. Peng } & \text { D. Wanigarathnajayasekara } & \text { K. Zhang } \\ \text { M. S. A. Perera } & \text { A. Wanniarachchi } & \text { S. Zhang } \\ \text { H. Pu } & \text { P. Wanniarachchige } & \text { X. Zhang } \\ \text { T. Rathnaweera } & \text { C. Wei } & \text { Y. Zhao } \\ \text { D. Rudakov } & \text { E. Westman } & \text { W. Zheng } \\ \text { M. Sassi } & \text { B. Wu } & \text { S. Zhi } \\ \text { Z. Shao } & \text { J. Wu } & \text { G. Zhu } \\ \text { W. Shen } & \text { W. Wu } & \text { J. Zhu }\end{array}$

We apologize for any errors or inadvertent omissions.

Publisher's Note Springer Nature remains neutral with regard to jurisdictional claims in published maps and institutional affiliations. 